\title{
Impact of Tunnel Groups on Pupil Diameter of Drivers on Mountainous Freeway in China: A Real-World Driving Study
}

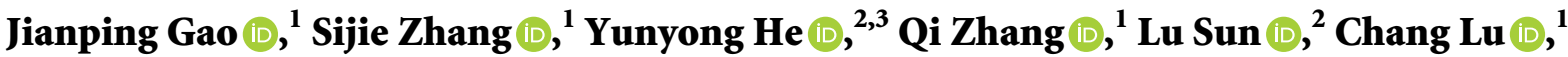 \\ Yi Wu $\mathbb{D},{ }^{1}$ Enhuai $\mathrm{He} \mathbb{D}^{2}{ }^{2}$ and Changfeng Yang $\mathbb{D}^{2}$ \\ ${ }^{1}$ School of Civil Engineering, Chongqing Jiaotong University, 66 Xuefu Avenue, Nan'an District, Chongqing 400074, China \\ ${ }^{2}$ Sichuan Highway Planning, Survey, Design and Research Institute Co., Ltd., Chengdu 610041, Sichuan, China \\ ${ }^{3}$ School of Civil Engineering, Southwest Jiaotong University, Chengdu 610041, Sichuan, China
}

Correspondence should be addressed to Jianping Gao; jianping-gao@163.com and Sijie Zhang; 897040253@qq.com

Received 1 July 2021; Revised 30 July 2021; Accepted 12 August 2021; Published 3 September 2021

Academic Editor: Jaeyoung Lee

Copyright ( 2021 Jianping Gao et al. This is an open access article distributed under the Creative Commons Attribution License, which permits unrestricted use, distribution, and reproduction in any medium, provided the original work is properly cited.

A real-world driving experiment was performed in the Wen-Ma section of the G4217 Rong-Chang Freeway situated in the Sichuan Province to investigate the impact law of the pupil diameter of drivers in tunnel groups on the mountainous freeway. The eye-movement data of drivers were collected, and the percentage of pupil diameter variable (PPDV) was used as a visual characteristic index. The analysis of the overall change in the PPDV of drivers in the experimental sections demonstrated that the PPDV in tunnel groups differed significantly between the nontunnel sections and single tunnel sections. Subsequently, a related model for the PPDV of drivers and the length of the connecting zone between tunnels was established, its reliability evaluated, and the smooth mutation value obtained on the basis of the mutation theory. Thereafter, a tunnel group definition standard based on the visual effect of drivers was developed. A six-zone approach was devised for the analysis of tunnel groups, and the result revealed that the different zones in the tunnel group have different impact on PPDV of drivers. The results revealed that the different zones of tunnel group have different impact on PPDV of drivers. Furthermore, lighting transition facilities should be set in the exit section of tunnel. The PPDV of drivers was negatively correlated with the length of the connecting zone of tunnel groups, and $100 \mathrm{~m}$ is the recommended safety length threshold for the connecting zone of tunnel groups.

\section{Introduction}

With the expansion of Chinese freeways to remote mountainous areas, the extent of mountainous freeways has considerably increased. Moreover, the proportion of tunnel sections on mountainous freeways has been increasing, which has led to the emergence of dangerous sections such as continuous tunnels or even multitunnel group sections [1]. The extreme climate, unsatisfactory safety facilities, and numerous tunnel sections make traffic safety in mountainous areas much more dangerous. In tunnel groups, drivers enter and exit tunnels several times in a short time, and the frequent changes in the illumination intensity lead to a repeated visual adaptation process between light and dark illuminations for drivers. It results in "black and white hole" effects and the deterioration of drivers' visibility. This seriously interferes with the visibility of drivers to obtain information in the driving task, thereby having seriously adverse effects on the safe operation of vehicles [2].

Repeated and rapid changes in the light environment require drivers to have extensive adaptability to the driving environment, which brings about more challenging driving conditions in tunnel groups [3]. Moreover, a long period of closed and narrow driving environment leads to safety risks owing to the uncertain driving behavior under the continuous psychological and physiological stresses of drivers $[4,5]$. Zhao et al. $[3,6]$ found that the visibility and vehicle speeds inside a tunnel were significantly different from those of single tunnel and tunnel group sections.

Previous studies have demonstrated that the connection segments within a tunnel group have a significant impact on the drivability [7]. Moreover, the risk degree of tunnel 
groups is higher than that of single tunnels, and the average crash rate of tunnel groups (1.72 per million VKT) [8] is greater than that of single tunnels (0.51 per million VKT) [9], which indicates that tunnel groups are riskier than single tunnels. Wang et al. [8] used the classification and regression tree (CART) model to study the influence of four factors, namely, the freeway alignment, driving behavior, vehicle characteristics, and environment, on the injury severity of crashes. They found that the driving behavior (i.e., human factor) was the most critical determinant of the injury severity of crashes on mountainous freeways. Since there exists a significant difference among the driving environment of the single and group tunnels; therefore, it is essential to conduct an in-depth research on how group tunnels sections affect drivers' behavior.

Firstly, the definition of tunnel groups should be clarified. At present, no specific definition standard for tunnel groups is available [10], although relevant research has been carried out in the industry. The studies have been focused on tunnel ventilation and lighting [7], traffic safety [10], fire safety [11], lane retention length and alignment, and the lighting adaptation process [12], etc. Yang and Guo [13] defined a tunnel group as two or more tunnels whose space is shorter than $1000 \mathrm{~m}$. There remains a lack of research on the definition of tunnel groups from the perspective of the physiological characteristics of drivers. Therefore, this study proposes a new definition of a tunnel group based on the pupil diameter of drivers.

Secondly, there were not many tunnel groups in the past due to the immature tunnel construction technologies. Since the recent decade, many tunnel groups have emerged in mountainous areas owing to the developed construction technologies. In the past, research on drivers' visual characteristics more focused on the extra-long tunnel [14] and other tunnel special sections such as entrance and exit sections [15]. Wang et al. [16] investigated drivers' visual characteristics under different radii and turning conditions in small-radius optically long tunnels, and they found that the smaller the tunnel radius, the more focused the gaze points on inside of the curves. Apart from this, some studies have been conducted to investigate the tunnels' traffic safety [17-19]. Pervez et al. [20] used seven-zone analysis method to analyze the accident safety of expressway long tunnel, and the results show that the crash rate increases firstly for the entrance area, then decreases in the mid zone, and increases again in the exit zone, and there are obvious differences in the causes of accidents in the above three zones. Similarly, Pervez et al. [21] also adopted a six-zone analysis approach to analyze the accident characteristics of freeway single tunnels of different lengths. Jung and Qin [22] have confirmed the strategies recommended quantitatively by KMOLIT through the random forest-based binomial regression with a data driven approach. Still, only a few studies have been done concentrating on driver visual characteristics in tunnel groups. These studies on tunnel groups have mainly focused on the crash distribution characteristics of different tunnel group sections [23], morphological statistics [8], exit and entrance safety $[3,24,25]$, and the safety evaluation of tunnel group sections [7]. As can be seen from the above, only a few studies have been conducted on the influence of human factors on truck drivers, and most of the research objects have been single tunnel groups. A single tunnel group is defined as only one tunnel group consisting of several single tunnels on the road section instead of several different tunnel groups like in the experimental road section.

In this study, to narrow the above research gaps and to consider the importance of drivers in driving safety, the Wen-Ma section of the G4217 Rong-Chang Freeway (WenMa Freeway), which has multiple tunnel groups, was used as the basis for a real-road experiment, and a mathematical statistical method was adopted for data analysis and comparison. This study proposes a definition of a tunnel group based on driving behavior and investigates the impact law of the tunnel group on driver's visibility using several mountainous freeways to provide a reference for the establishment of traffic safety facilities and the traffic management of tunnel group sections.

\section{Materials and Methods}

2.1. Experimental Road Section. The real-world driving experiments were performed during the daytime from November 12 to 14,2020 . The road section selected for this study is a part of the Wen-Ma Freeway with a $69.97 \mathrm{~km}$ length situated in the Sichuan Province, China. The experimental road section has a four-lane dual carriageway, and the design speed is $80 \mathrm{~km} / \mathrm{h}$. According to Specifications for Design of Highway Tunnels Section (JTG/T D70/2-2014) [26] of China, road tunnels can be divided into the following four categories: short tunnels $(L<500 \mathrm{~m})$, medium tunnels (500 $\mathrm{m} \leq L<1000 \mathrm{~m})$, long tunnels $(1000 \mathrm{~m} \leq L<3000 \mathrm{~m})$, and extra-long tunnels $(L>3000 \mathrm{~m})$. There are 18 tunnels in the experimental road section, including four short tunnels, ten long tunnels, and four extra-long tunnels. The length of the tunnels accounted for $54.6 \%$ of the experimental road section. The distribution of the tunnel spacing is summarized in Table 1, and the tunnel distribution is shown in Figure 1 .

2.2. Experimental Drivers and Vehicles. Three healthy drivers with no driving experience on Wen-Ma Freeway, without major crash experience, and with more than 10 years of driving experience were selected for the eye-movement data collection, as shown in Figure 2. The participants drove their vehicles on the experimental section successively under the same driving conditions, and the drivers did not suffer from fatigue or drink alcohol during the experiment. A researcher was assigned to record the road conditions and distribution of major structures. A four-axle truck was selected for the experimental model.

2.3. Experimental Instruments. A Smart Eye noncontact eye tracker with a collection frequency of $60 \mathrm{~Hz}$ was used as the main data acquisition device to record the eye-movement data of the drivers, such as the pupil diameter, eyelid opening, fixation, saccade, and blinking. Moreover, the vehicle was equipped with an in-car eye-movement 
TABLE 1: Distribution of tunnels.

\begin{tabular}{|c|c|c|c|c|}
\hline Serial number & Name of tunnel & Length $(\mathrm{m})$ & Tunnel type & Spacing distance $(\mathrm{m})$ \\
\hline 1 & Pengjiaheba Tunnel & 117 & Short tunnel & - \\
\hline 2 & Jianyangping Tunnel No. 2 & 1636 & Long tunnel & 2840 \\
\hline 3 & Jianyangping Tunnel No. 1 & 1420 & Long tunnel & 97 \\
\hline 4 & Putou Tunnel & 2348 & Extra-long tunnel & 125 \\
\hline 5 & Lixian Tunnel & 5500 & Extra-long tunnel & 6272 \\
\hline 6 & Weiguan Tunnel & 1159 & Long tunnel & 306 \\
\hline 7 & Ganbao Tunnel & 4582 & Extra-long tunnel & 622 \\
\hline 8 & Mudui Tunnel & 2342 & Long tunnel & 1929 \\
\hline 9 & Puxigou Tunnel & 394 & Short tunnel & 80 \\
\hline 10 & Xindianzi Tunnel & 1871 & Long tunnel & 305 \\
\hline 11 & Xuecheng Tunnel No. 2 & 185 & Short tunnel & 3112 \\
\hline 12 & Xuecheng Tunnel No. 1 & 3463 & Extra-long tunnel & 216 \\
\hline 13 & Gucheng Tunnel & 2911 & Long tunnel & 342 \\
\hline 14 & Tonghua Tunnel No. 2 & 3025 & Extra-long tunnel & 152 \\
\hline 15 & Tonghua Tunnel No. 1 & 2475 & Long tunnel & 105 \\
\hline 16 & Taoping Tunnel & 2374 & Long tunnel & 818 \\
\hline 17 & Xiazhuang Tunnel & 215 & Short tunnel & 6388 \\
\hline 18 & Sangping Tunnel & 2181 & Long tunnel & 1656 \\
\hline
\end{tabular}

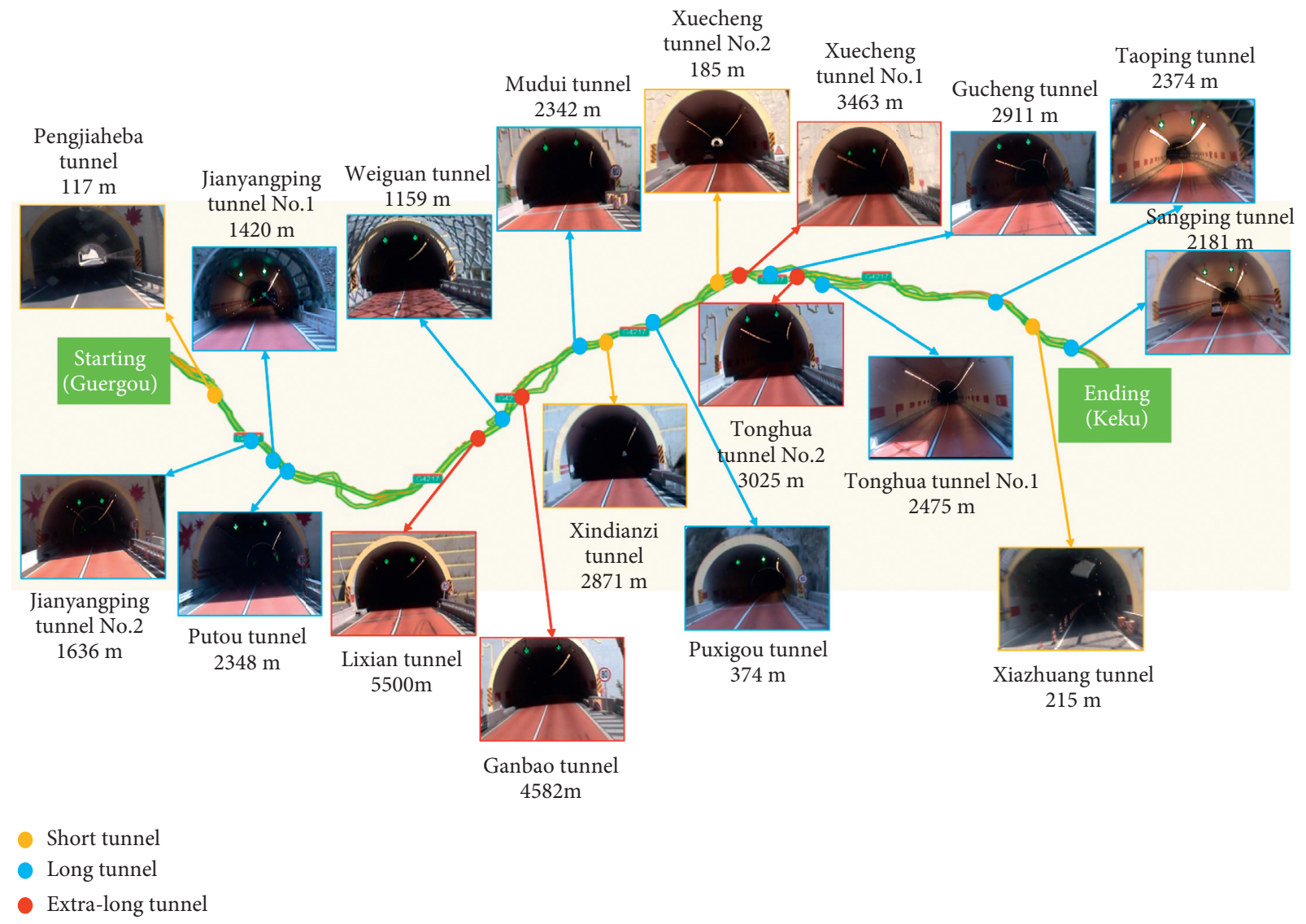

Figure 1: Tunnel distribution in the experimental section.

capturing camera, and a scene camera was placed outside the vehicle to record the driving environment along the road.

2.4. Experimental Indicators. The most notable difference in the driving environment between a tunnel section and a nontunnel section concerns the change in the light environment, whereas that between a tunnel group section and a single tunnel section concerns the repetition of the light change. A driver enters and exits tunnels repeatedly within a short time, and the driver experiences the process of light and shade adaptation [27]. The pupil change of the driver exhibits the most sensitive and intuitive reflection of 

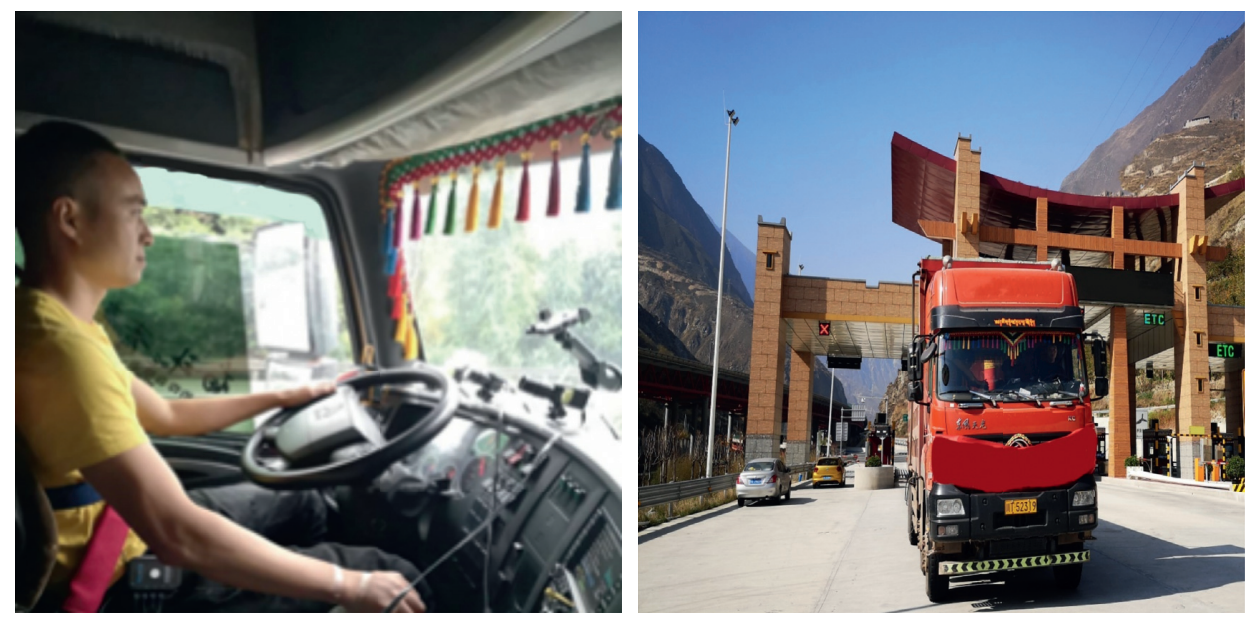

Figure 2: Experimental drivers and vehicles.

the repeatedly changing driving environment between tunnels. Because of the differences among drivers, pupil diameter was used as the experimental indicator in this study. On the basis of a relevant prior study [28], the pupil diameter of the driver in the static state was used as the reference value while the percentage of pupil diameter variable (PPDV) was used as the pupil change indicator. The PPDV was calculated as shown in the following equation:

$$
\operatorname{PPDV}=\left|\frac{\left(\left(d_{i}-d_{0}\right) / d_{0}\right)}{\triangle t} \times 100 \%\right|,
$$

where $d_{0}$ is the baseline value of the pupil diameter of the driver $(\mathrm{mm}), d_{i}$ is the pupil diameter of the driver when driving at time $i(\mathrm{~mm})$, and $\Delta t$ is the time interval (s).

Because the driving environment in the experiment was generally the same, the percentage weighted average of the pupil diameter variable of the three drivers was used for analysis.

$$
\operatorname{PPDV}_{m}=\frac{\sum_{i=1}^{n} \operatorname{PPDV}_{i}}{n},
$$

where $\mathrm{PPDV}_{m}$ is the percentage weighted mean of the driver pupil diameter variable $(\mathrm{mm}), \mathrm{PPDV}_{i}$ is the PPDV at time $i$ $(\mathrm{mm})$, and $n$ is the number of drivers.

\section{Results}

3.1. Data. The pupil diameters of the three drivers in the static state were measured, and the arithmetic mean value was used as the reference value for the pupil diameter. The PPDV was calculated according to equations (1) and (2), and the mean value was taken as the feature point every $1 \mathrm{~s}$. To ensure the validity of the data, pupil diameter tracking quality data, which were provided by the eye tracker, with values of less than 0.5 , were removed $[28,29]$.

On the basis of previously reported findings [30], the experimental sections were preliminarily divided into three categories based on the different tunnel spacing as follows: (1) the continuous tunnel section, which included two or more tunnels with an interval of less than $1000 \mathrm{~m}$; (2) the single tunnel section, which consisted of an independent tunnel with an interval of more than $1000 \mathrm{~m}$ from other tunnels; (3) the nontunnel section, which included the remainder of the experimental road section. The Kolmogorov-Smirnov test results of the PPDV of the drivers in the three road sections are presented in Table 2.

The test results demonstrated that the PPDV in the nontunnel and continuous tunnel sections approximately conformed to a normal distribution, whereas the PPDV in the single tunnel section conformed to a normal distribution at $\alpha=0.05$. The Grubbs test method was used to screen and eliminate the outliers of the PPDV in the three sections; the results for the nontunnel sections are shown in Figure 3. This test method is suitable for upper, lower, and bilateral cases. As the sample data were processed by absolute values, the Grubbs upper situation test was adopted to eliminate the outliers in this study.

As the experiment lasted for more than $60 \mathrm{~min}$, to demonstrate the overall change in the PPDV, the average value was used as the feature point every $10 \mathrm{~s}$ to obtain the overall distribution of the PPDV in the test section for analysis, as illustrated in Figure 4.

One-way analysis of variance (ANOVA) was performed on the PPDV in the three sections. The continuous tunnel section exhibited a significant difference from the nontunnel and single tunnel sections, as indicated in Figure 5.

It can be observed from Figures 4 and 5 that the PPDV exhibited different characteristics in different sections, as follows: (1) In the nontunnel section, the mean PPDV was $16.70 \%$, whereas the standard deviation was $15.56 \%$, which was the smallest among the three sections, indicating that the percentage distribution was the most stable when the drivers were driving in the nontunnel section. (2) In the continuous tunnel section, the average PPDV of the drivers reached $40.69 \%$, whereas the standard deviation was $24.66 \%$, which was the largest among the three different sections, indicating that when the drivers were driving in the continuous tunnel section, the percentage distribution was uneven and fluctuated significantly. (3) In the single tunnel section, the 
TABLE 2: Kolmogorov-Smirnov test results for three types of road sections.

\begin{tabular}{lccc}
\hline Road section & Kolmogorov-Smirnov Z & $P$ (concomitant probability) & $\mu(\mathrm{mean})$ \\
\hline Continuous tunnel & 0.12 & 0.04598 & 0.4325 \\
Single tunnel & 0.12 & 0.09486 & 0.2857 \\
Nontunnel & 0.11 & 0.04972 & 0.2253 \\
\hline
\end{tabular}

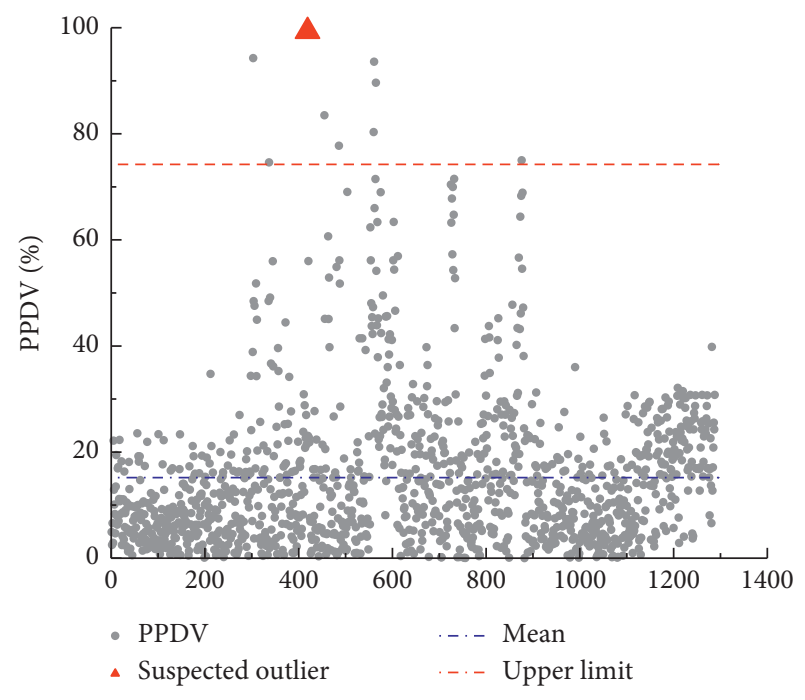

Figure 3: Results of Grubbs test (nontunnel section).

average PPDV was $28.57 \%$, whereas the standard deviation was $18.38 \%$, indicating that although the driver pupil diameter fluctuated compared with that of the nontunnel section when driving in the single tunnel section, it was more stable compared with that of the continuous tunnel section.

The reasons for the different variation characteristics are as follows: (1) The nontunnel section was in a naturalistic driving environment with high and continuous illuminance, and it had low psychological pressure and stable percentage distribution of the pupil diameter variable [4]. (2) In the continuous tunnel section, the sudden change in illumination at the entrance and exit of the tunnel as well as the repeated occurrence of different tunnels directly affected the physiological changes of the driver [31,32]; thus, the psychological pressure of the driver increased, resulting in an unstable and large fluctuation in the distribution of the PPDV. (3) In the single tunnel section, although there was a sudden change in illumination at the entrance and exit of the tunnel, there was no repeated change in illumination [32]. Therefore, although the distribution of the PPDV in this section was not stable, the fluctuation level of the corresponding indicator was lower than that of the continuous tunnel section.

\subsection{Classification and Influence Range of Tunnel Groups Based on Driver Vision}

3.2.1. Classification of Tunnel Groups Based on Driver Vision. As illustrated in Figure 4, when the distance between two tunnels was comparatively short, the PPDV of the drivers between the two tunnels always remained at the level of the tunnel driving environment. When the distance between the two tunnels was comparatively long, the PPDV of the drivers gradually decreased and no longer exhibited tunnel section characteristics.

To describe the relationship between the PPDV and tunnel spacing objectively, a nonlinear curve fitting analysis was conducted for both, as depicted in Figure 6. The results of the variance analysis are presented in Table 3.

The evaluation coefficient of the fitting curve between the PPDV and distance between tunnels was $R^{2}=0.9656$, $p \leq 0.001$, which exhibited a good degree of fitting and high reliability. The relationship between the two can be expressed as follows:

$$
\operatorname{PPDV}_{m}=0.1641+0.3452 \exp ^{(-x / 394)},
$$

where $\mathrm{PPDV}_{m}$ is the percentage weighted mean of the PPDV $(\mathrm{mm})$ and $x$ is the tunnel spacing $(\mathrm{m})$.

According to the aforementioned analysis, there were evident differences among the nontunnel, continuous tunnel, and single tunnel sections, which could represent the change in the PPDV under normal driving conditions when driving in the nontunnel section. To determine the threshold of tunnel spacing as the standard for the tunnel group classification, it was necessary to select a sudden characteristic value [33] from the nontunnel section as the critical value of the three sections. In other words, this value was used to distinguish whether the driver was in a continuous or single tunnel section when driving on the test section. If the PPDV was higher than the sudden change value, the driver could be considered to be in a continuous or single tunnel section; otherwise, the driver could be considered to be in a nontunnel section. According to Figure 6 and equation (3), the relational model function was a monotone function, and the corresponding value of the inflection point could not be obtained. Considering the behavior adaptation of dangerous sections when driving in nontunnel sections, drivers react to the outside world because of the changes in the roadside environment or alignment, which leads to a sudden change in the PPDV. Therefore, the cumulative frequency statistics of the pupil mass percentage of the driver driving in the nontunnel section were determined, and in combination with the catastrophe theory, the eigenvalues were calculated. The percentile distribution of the PPDV in the nontunnel section is depicted in Figure 7.

Based on the work of Liu et al. [29], Figure 7 was analyzed using the Zeeman heartbeat model. As shown in Figure 7 , there was a smooth mutation point. The corresponding value of the mutation point was $22.27 \%$, which was the eigenvalue of the driver in the nontunnel section. This value reflected the critical value of the percentage fluctuation in the PPDV from the nontunnel section to the continuous 


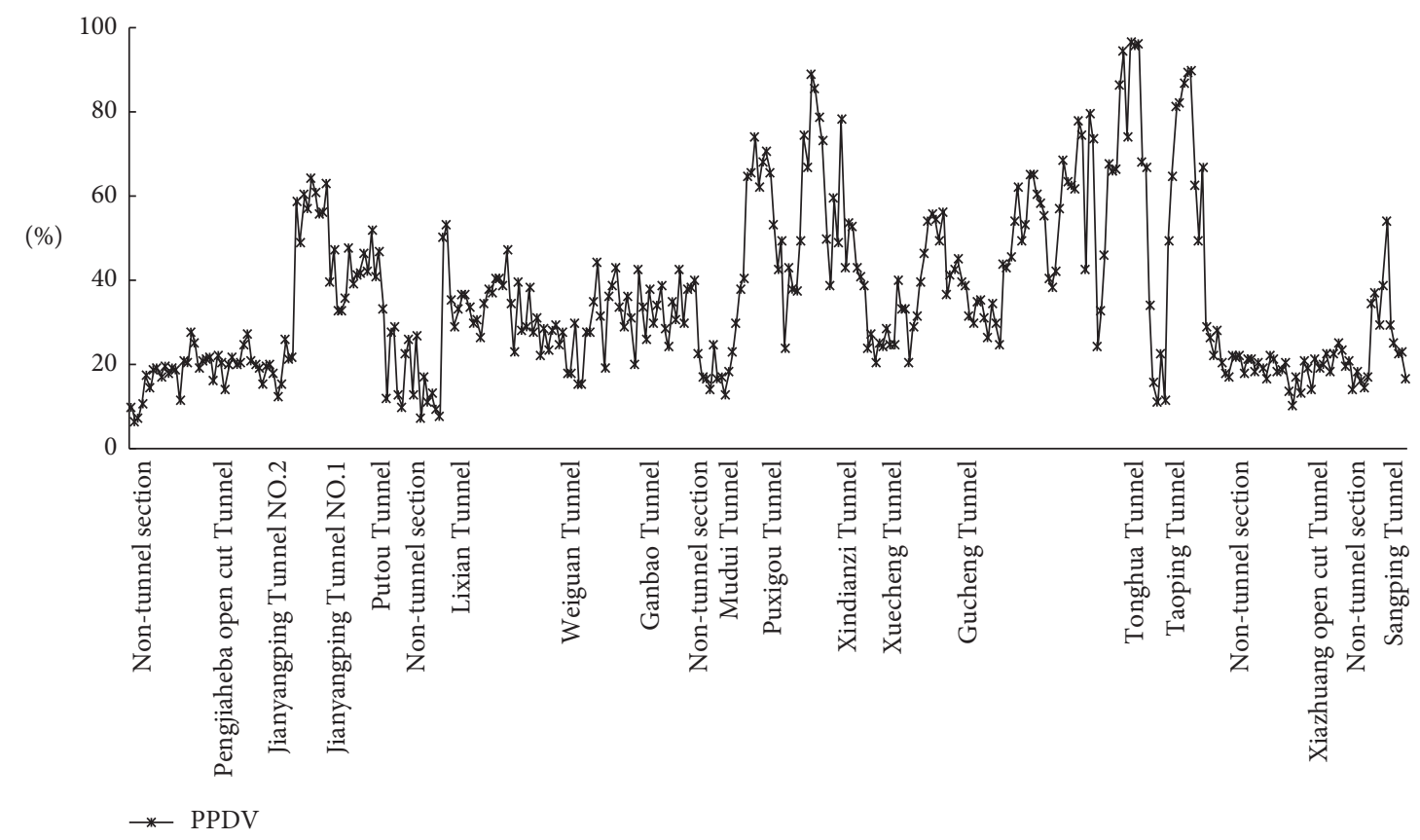

FIgURE 4: Overall change distribution of PPDV.

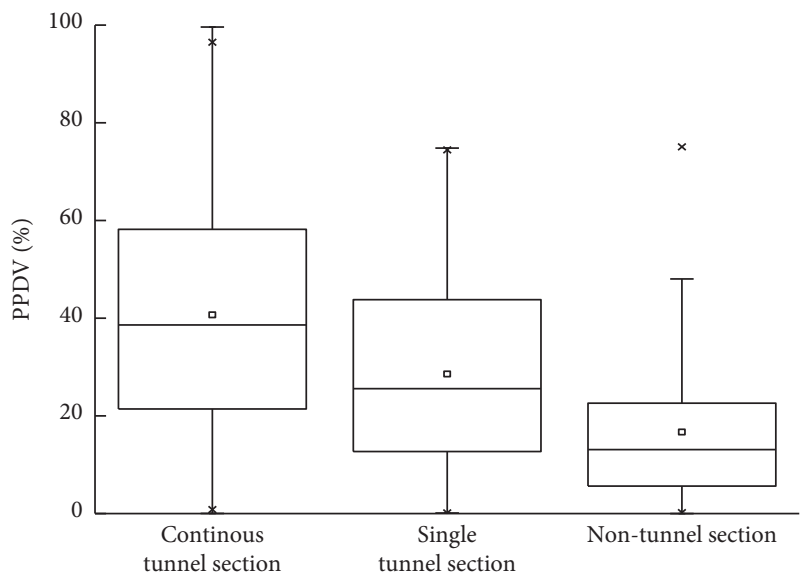

Figure 5: One-way ANOVA of three different road sections.

tunnel section, which could be used as the reference value of the general road section. The value $22.27 \%$ was substituted into the relational model for regression. The corresponding critical value of the tunnel spacing was $697.14 \mathrm{~m}$, which was rounded off to $700 \mathrm{~m}$.

Therefore, the tunnel group definition standard based on the pupil change of the driver is that three or more adjacent tunnels should be treated as a group of tunnels when the spacing between these is less than $700 \mathrm{~m}$.

In the experiments performed in this study, tunnels were divided into groups according to the criteria specified in the earlier definition, and the results are presented in Table 4.

3.2.2. Analysis of Influence Range of Tunnel Groups. The tunnel has an impact on visibility indicators of the driver before entering the tunnel, and it lasted some distance from

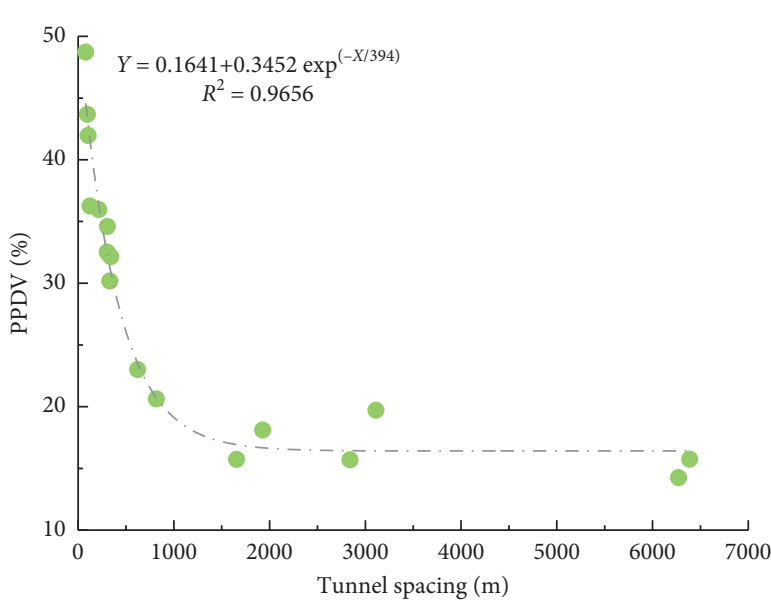

FIGURE 6: Relationship between PPDV and tunnel spacing.

the exit of the tunnel $[14,34]$. Therefore, it is necessary to determine the influence range of tunnel groups in this study.

Figure 8 shows the PPDV distributions of the drivers at the first tunnel entrance section and the final tunnel-exit section of the four tunnel groups.

Figure 8 demonstrates the following:

(1) Before driving into the tunnel group, the PPDV of the driver started to exceed the smooth mutation value and exhibited a slow growth trend until driving into the tunnel group, when the PPDV increased sharply. Thereafter, the growth range was relatively stable and remained at a high level for a period after entering the tunnel group. The analysis results indicate that the psychological pressure of the driver increased when they saw the tunnel group signs and 
TABLE 3: Model variance analysis results of the relationship between PPDV and tunnel spacing.

\begin{tabular}{lcccr}
\hline Source of variance & Degree of freedom & Quadratic sum & Mean square & $F$ \\
\hline Regression & 2 & 0.1900 & 0.0950 & 178.260 \\
Residuals & 14 & 0.0075 & 0.0005 & $\leq 0.001$ \\
Unmodified total & 17 & 1.5465 & & \\
\hline
\end{tabular}

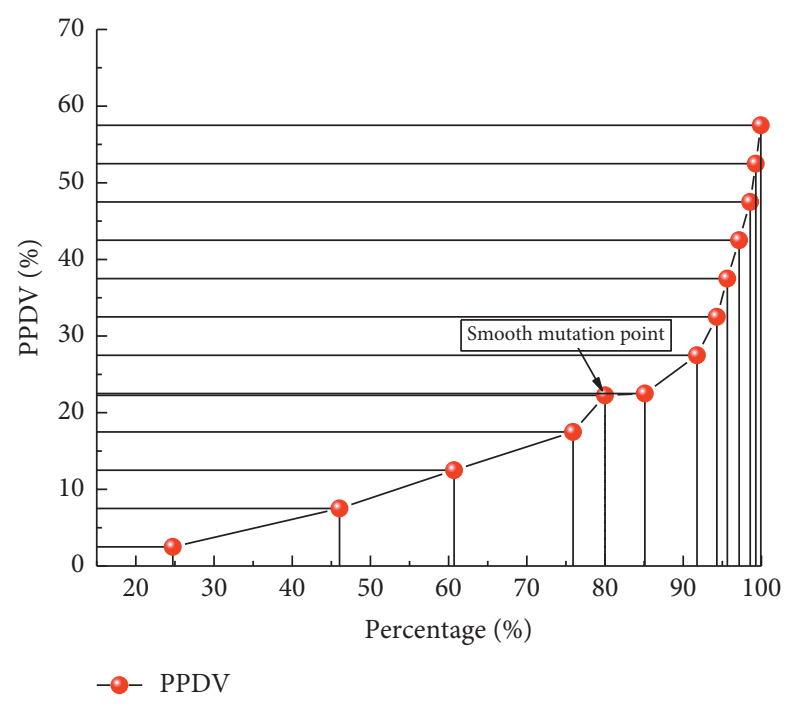

FIgURe 7: Percentage distribution of PPDV in nontunnel section.

TABle 4: Tunnel group division.

\begin{tabular}{|c|c|c|c|c|}
\hline Serial number & Tunnel group & Name & $\begin{array}{l}\text { Length } \\
(\mathrm{m})\end{array}$ & $\begin{array}{c}\text { Spacing with } \\
\text { upstream tunnel }(\mathrm{m})\end{array}$ \\
\hline \multirow{3}{*}{1} & \multirow{3}{*}{ Jianyangping Tunnel group (ZK113 + 398 ZK119 + 024) } & Jianyangping Tunnel No. 2 & 1636 & - \\
\hline & & Jianyangping Tunnel No. 1 & 1420 & 97 \\
\hline & & Putou Tunnel & 2348 & 125 \\
\hline \multirow{3}{*}{2} & \multirow{3}{*}{ Lixian-Gambao Tunnel group (ZK94 + 861 ZK107 + 126) } & Lixian Tunnel & 5500 & - \\
\hline & & Weiguan Tunnel & 1159 & 306 \\
\hline & & Ganbao Tunnel & 4582 & 622 \\
\hline \multirow{3}{*}{3} & \multirow{3}{*}{ Mudui-Xindianzi Tunnel group (ZK86 + 940 ZK92 + 932) } & Mudui Tunnel & 2342 & - \\
\hline & & Puxitgou Tunnel & 394 & 80 \\
\hline & & Xindianzi Tunnel & 1871 & 305 \\
\hline \multirow{5}{*}{4} & \multirow{5}{*}{ Xuecheng-Tonghua tunnel group (ZK70 + 100 ZK83 + 828) } & Xuecheng Tunnel No. 2 & 185 & - \\
\hline & & Xuecheng Tunnel No. 1 & 3463 & 216 \\
\hline & & Gucheng Tunnel & 2911 & 342 \\
\hline & & Tonghua Tunnel No. 2 & 3025 & 332 \\
\hline & & Tonghua Tunnel No. 1 & 2475 & 105 \\
\hline
\end{tabular}

structures ahead. Therefore, the PPDV of the driver increased slowly when the driver did not drive into the tunnel group. When driving into the tunnel group, the difference in the tunnel environment led to a sudden increase in the PPDV. After driving into the tunnel group for seconds, the driver adapted to the driving environment, and the PPDV exhibited a relatively stable state, which was higher than the smooth mutation value.
(2) Approximately 10 seconds (Jianyangping Tunnel group: 10 seconds; Lixian Tunnel group:10 seconds; Mudui Tunnel group: 9 seconds; Xuecheng Tunnel group: 9 seconds) before entering the tunnel group, the PPDV of the driver fluctuated significantly between $15 \%$ and $23 \%$. The PPDV of the driver suddenly changed when driving out of the tunnel group, and it gradually decreased to the average level for the general road section for seconds thereafter. This is because there was no 


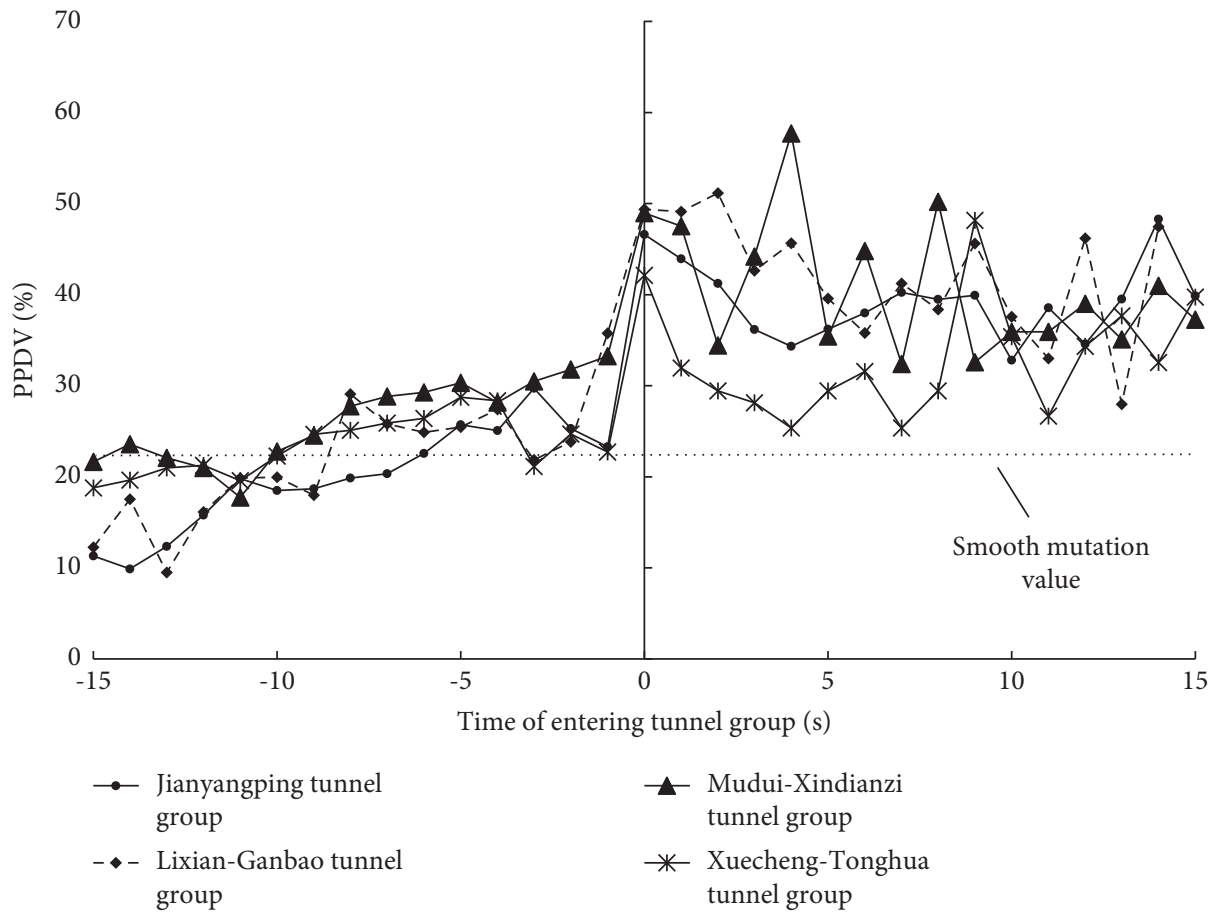

(a)

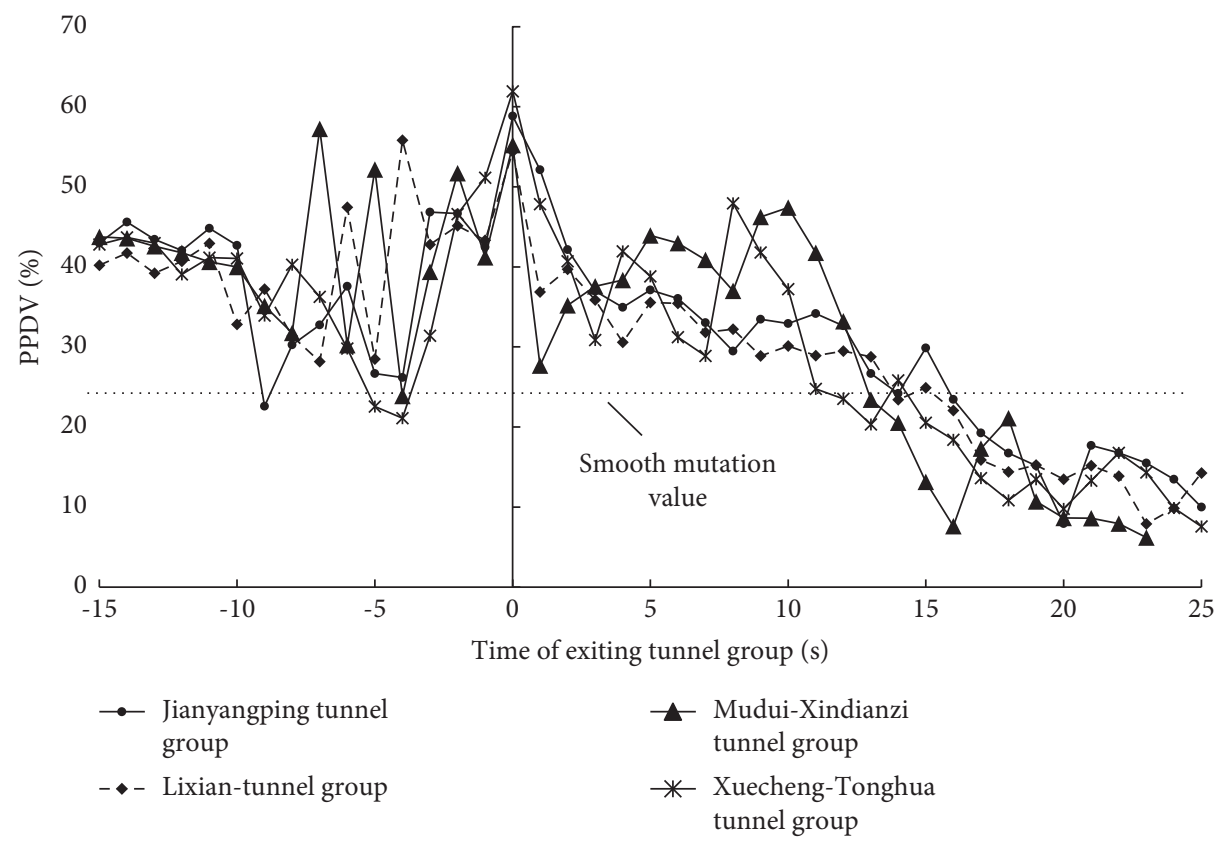

(b)

Figure 8: Analysis of influence range of tunnel groups. (a) Entrances of different tunnel groups. (b) Exits of different tunnel groups.

lighting transition section at the exit of the tunnel, and the white hole effect was evident, leading to a large fluctuation in the PPDV.
(3) Fifteen seconds (Jianyangping Tunnel group: 13 seconds; Lixian tunnel group: 15 seconds; Mudui tunnel group: 14 seconds; Xuecheng tunnel group: 
14 seconds) after exiting the tunnel group, the PPDV of the driver gradually decreased to the average level for the general section. This is because the driver had to complete the adaptive process within 3-5 seconds after driving out of the tunnel group. Following the Ming adaptation, the driver adjusted the driver's own state and stabilized the mood, and the PPDV gradually decreased and fell below the smooth mutation value.

The results of the above analysis reveal that the tunnel group affects the PPDV of drivers 10 seconds before entering and 15 seconds after exiting the tunnel group.

Therefore, the driving distance of $225 \mathrm{~m}$ before entering the tunnel group till $335 \mathrm{~m}$ after exiting the tunnel group, with a tunnel speed limit of $80 \mathrm{~km} / \mathrm{h}$, was determined as the influence range of the tunnel group on the PPDV of drivers.

\subsection{Pupil Diameter Distribution of Drivers in Tunnel Groups}

3.3.1. Analysis of Different Sections of Tunnel Groups. The Chinese Specifications for Design of Highway Tunnels Section (JTG/T D70/2-2014) [26] claimed that the single tunnel can be divided into entrance zone, transition zone, and exit zone base on different lighting requirements. Considering specific characteristics of tunnel group and combining with the zone division of a single tunnel and the aforementioned analysis results of Section 3.2.2, the access zone, the removed zone, and the connecting zone should be regarded as the zones of the tunnel group. As illustrated in Figure 9, the tunnel group is divided into six zones.

Six zones are defined as follows:

(i) Access zone: this part is the nontunnel road section in front of the first tunnel portal in the tunnel group. The analysis results of Section 3.2.2 show that the driver's PPDV has been impacted by the tunnel group $225 \mathrm{~m}$ before entering the first tunnel portal of the tunnel group; the length of access zone is identified as $225 \mathrm{~m}$ in this study.

(ii) Removed zone: this part includes the nontunnel section starting from the exit of the last tunnel in the tunnel group. Similarly, according to the results of tunnel group influence zone analysis, the length of this zone is identified as $335 \mathrm{~m}$.

(iii) Entrance zone: this zone starts at the portal of the tunnel. According to the design data and experimental video of Wen-Ma Freeway, the tunnel entrance zone of the experimental zone is equipped with a $200 \mathrm{~m}$ lighting transition section to reduce the white hole effect. Thus, the length of this zone is identified as $200 \mathrm{~m}$.

(iv) Exit zone: for the sake of analysis, the length of this zone is the same as that of the entrance zone, including $200 \mathrm{~m}$ in front of the tunnel exit.

(v) Connecting zone: this zone includes the part between two adjacent tunnels in the tunnel group. (vi) Adaptive zone: this zone constitutes the remainder of the tunnel.

The PPDV of the drivers in the six zones of the tunnel groups is presented in Figures 10-13.

The general law of the pupil changes of the driver when driving within the various zones of the tunnel group could be obtained based on the analysis results of the tunnel group zones. The PPDV increased in the entrance zone; then, in the adaptive zone, the PPDV was steady and had a slow growth state. It subsequently decreased in the exit zone and then decreased in the connecting zone. After entering the downstream tunnel, this process was repeated until exiting from the final tunnel of the tunnel group.

The PPDV of the driver increased closer to the level of the tunnel entrance section when driving into the access zone. In addition to the difficulty of the driver to obtain accurate driving information in the tunnel through vision prior to entering the tunnel, the driver will also see the relevant signs of the tunnel groups before entering them, resulting in psychological pressure on the driver. In the entrance zone, the PPDV increased gradually with the increase in the distance into the tunnels; and there was a lighting transition section at the entrance of the tunnels. Therefore, the growth rate was relatively slow. In the adaptive zone, the PPDV increased gradually from the entrance zone to the maximum value. In the exit zone, the drivers experienced repeated environmental light and shade changes, resulting in repeated visual fatigue and reduced visual acuity, which led to visual retardation when driving in the exit zone. It is found that there was no lighting transition section at the exit zones of the tunnel groups and the average level (31.44\%) of the exit zones of the tunnel groups was higher than that $(34.30 \%)$ of the entrance zones, which indicates that the lighting transition section should also be set at the exit of the tunnel to effectively control the fluctuation range of the pupil diameter of drivers at tunnel exits. In the removed zone of the tunnel groups, the driver had passed through three or more tunnels consecutively and had adapted physically and mentally to the continuous changes in the driving environment. Thus, the PPDV was reduced in the removed zone.

Moreover, according to the identification of the length of the connecting zone, the law of changes in the pupil diameter of the driver when driving in the connecting zone was significantly affected by the length of the connecting zone; the specific influence law will be analyzed further later.

\subsubsection{Analysis of PPDV of Tunnel Group Connecting Zone.} A correlation analysis was conducted between the PPDV and the length of the connecting zone between tunnels. The analysis results are presented in Table 5 .

At a significance level of $\alpha=0.05$, the correlation analysis results demonstrated that the statistical significance was $P=0.010<0.050$, and the coefficient was 0.764 , indicating that the connecting zone length was strongly correlated with the PPDV. Further analysis was performed on the influence rule of the PPDV of the drivers with the length of the connecting zone, as illustrated in Figure 14. 


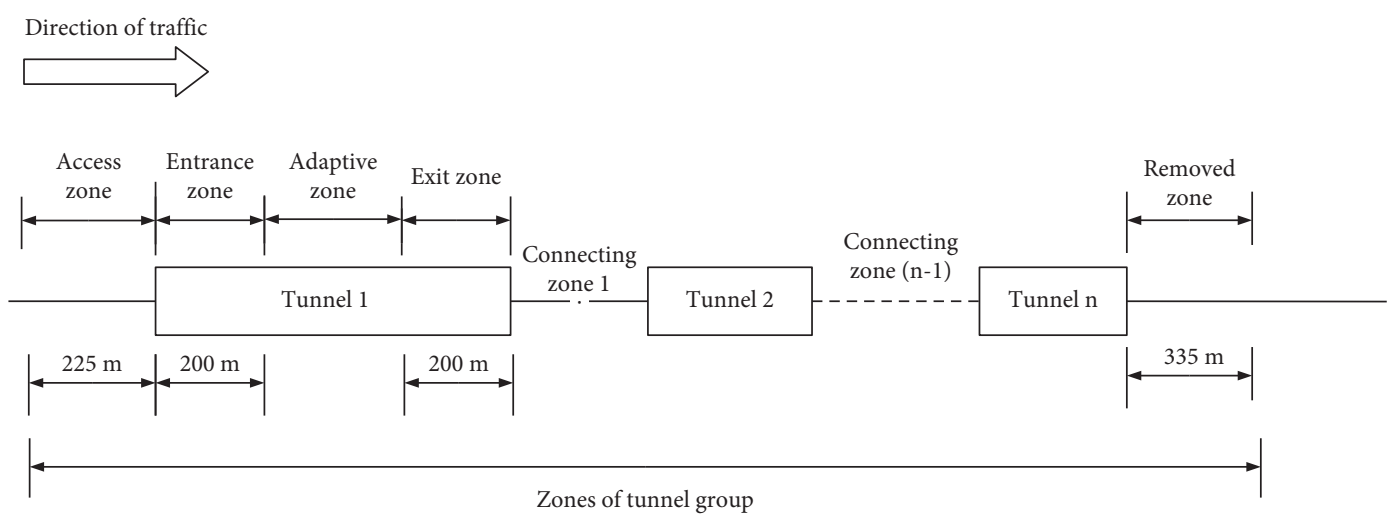

Figure 9: Section divisions of tunnel groups.

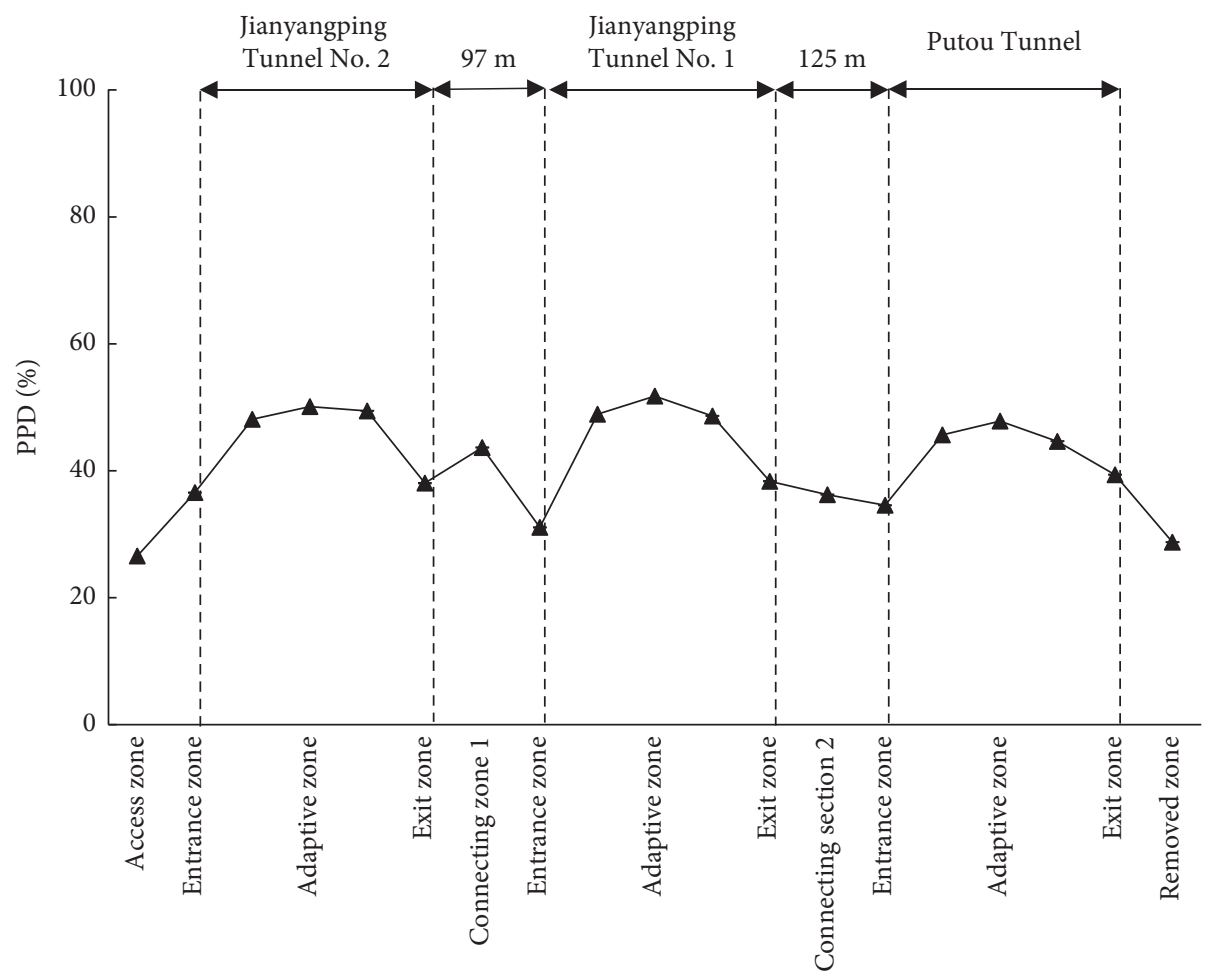

Figure 10: Zone analysis of Jianyangping Tunnel group.

As can be observed from Figure 14, the PPDV of the drivers was negatively correlated with the connecting zone lengths. A shorter connecting zone resulted in a higher PPDV and vice versa. This demonstrated that shorter connecting zones led to a shorter adaptive distance that remained for the driver. If the distance was too short, the driver would not have sufficient adaptive time to complete the adaptation prior to entering the next tunnel; thus, the PPDV remained the same or even increased compared with the level of pupil change in the tunnel.

To improve the driver adaptability to the driving environment in a short connecting zone, it is necessary to determine the safety critical length of the connecting zone of tunnel groups and propose safety guarantee suggestions accordingly.
The percentile distribution statistics of the PPDV of drivers in the connecting zones of four tunnel groups were determined, and $85 \%$ quantiles $[27,28]$ were selected as eigenvalues to be substituted into equation (3) for calculation. The PPDV was determined as $43.30 \%$, which was substituted into the model to calculate the corresponding value of $98.21 \mathrm{~m}$ that was rounded to $100 \mathrm{~m}$. Therefore, $100 \mathrm{~m}$ was considered as the safety critical value to guide the establishment of safety facilities for tunnel groups.

To ensure the continuity of the driving environment, according to the critical value of the safety length of the connecting zones of the tunnel groups, it is suggested to establish environmental improvement facilities in the entire connecting zones when the length $\left(L_{C}\right)$ of the connecting zone is less than or equal to $100 \mathrm{~m}$. When $L_{C}>100 \mathrm{~m}$, it is 


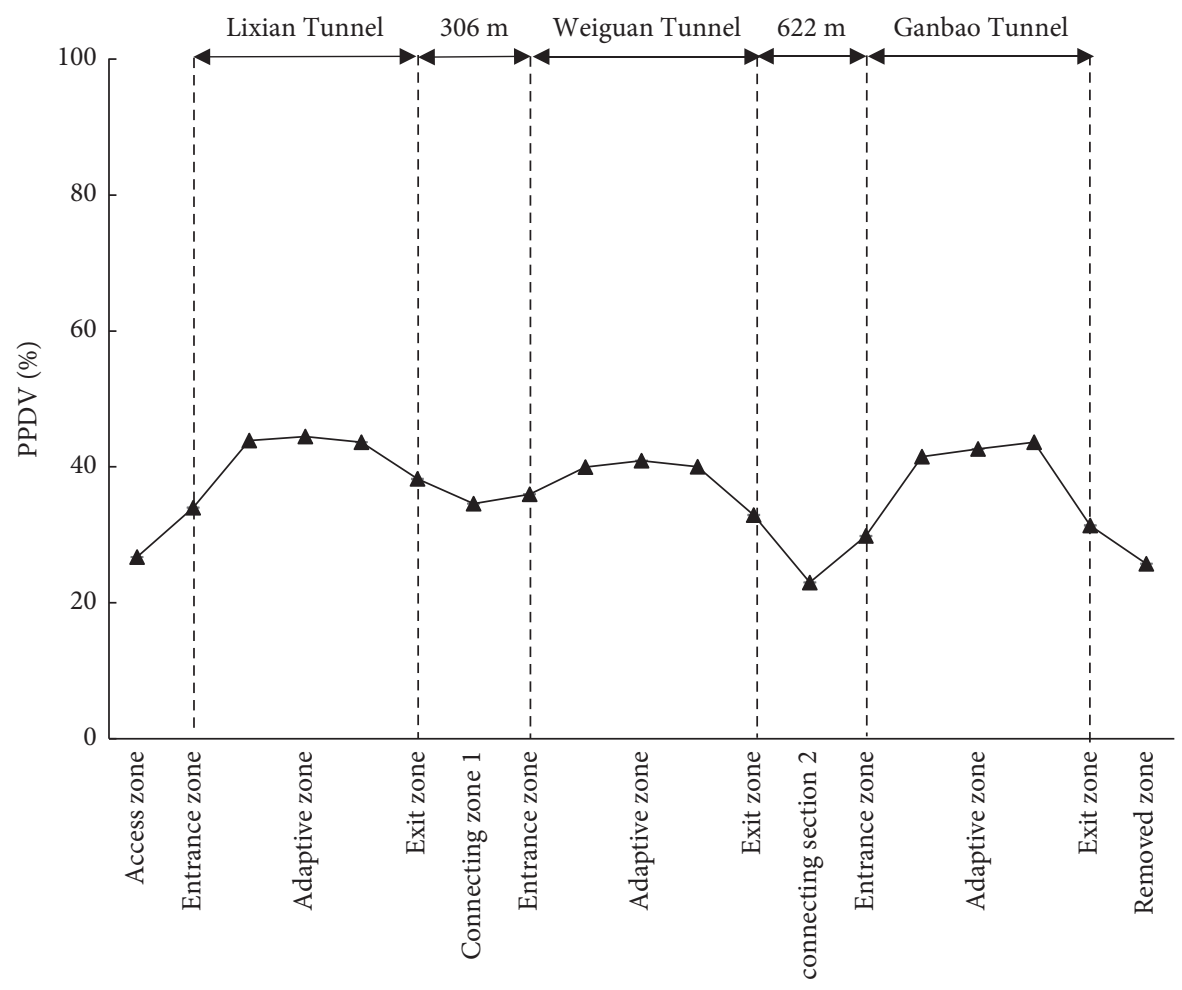

FIgURE 11: Zone analysis of Lixian-Ganbao Tunnel group.

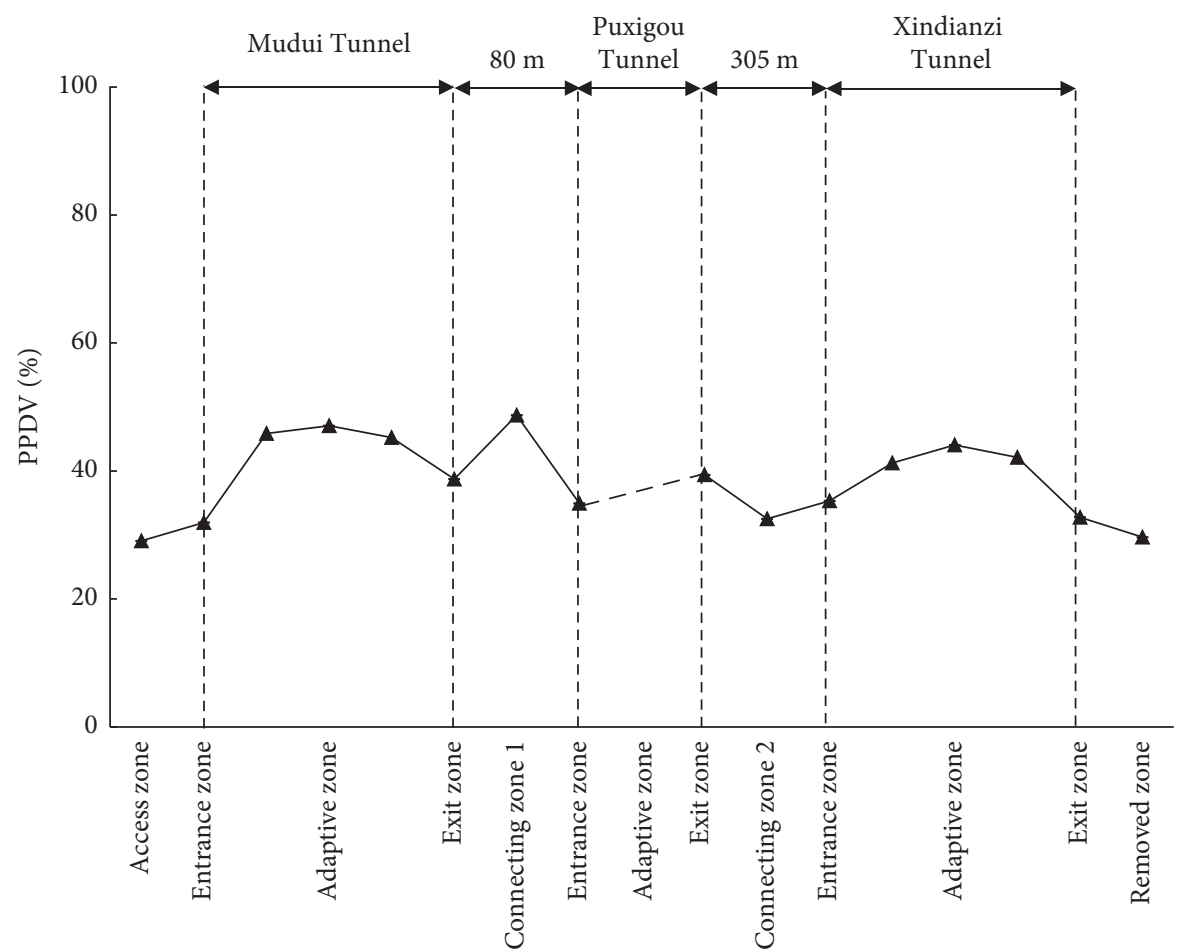

Figure 12: Zone analysis of Mudui-Xindianzi Tunnel group. 


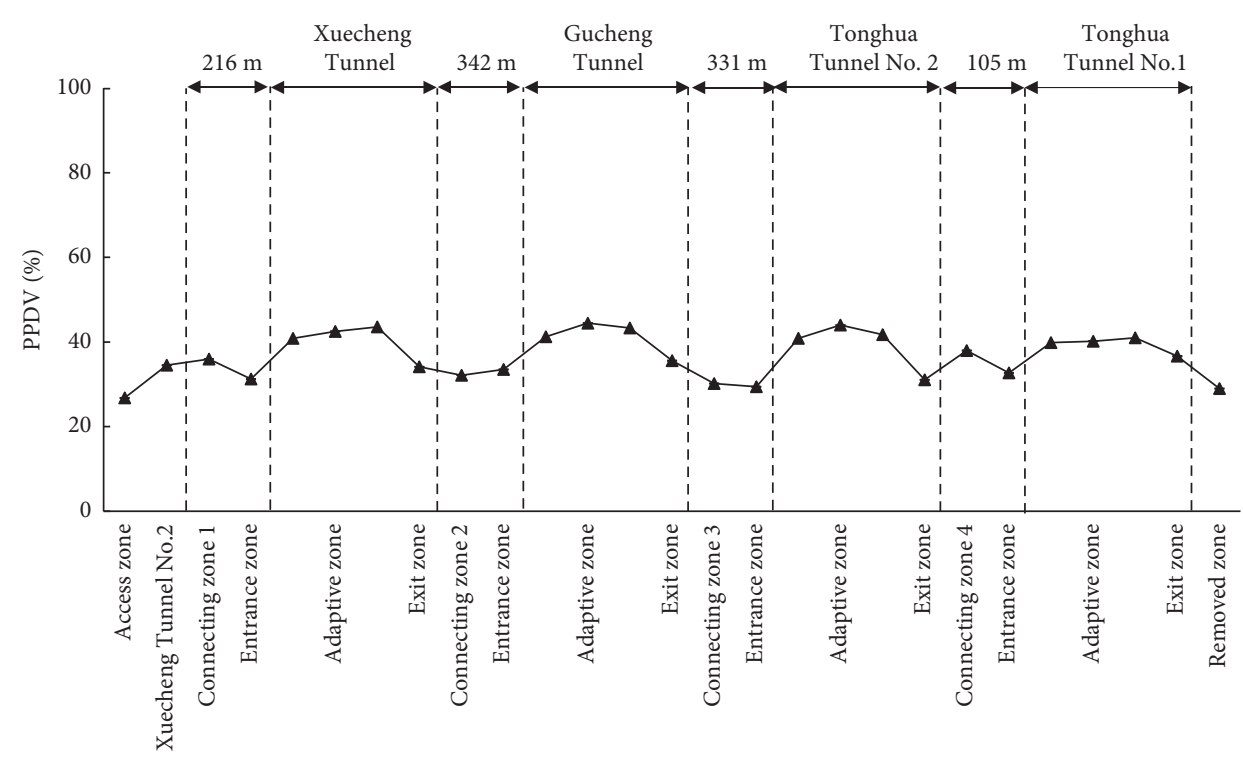

Figure 13: Zone analysis of Xuecheng-Tonghua Tunnel group.

TABLe 5: Percentage correlation analysis of connecting zone length $\left(L_{C}\right)$ and PPDV.

\begin{tabular}{lcc}
\hline & & Length $(\mathrm{m})$ \\
\hline \multirow{2}{*}{$\operatorname{PPDV}(\%)$} & Coefficient & Sig. \\
& -0.764 & 0.010 \\
\hline
\end{tabular}

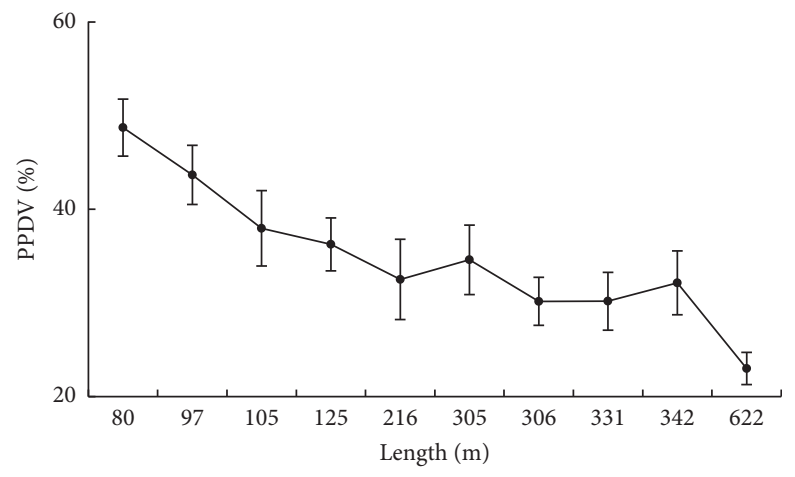

FIgURE 14: Change analysis of PPDV and different connecting zone lengths.

recommended to establish environmental improvement facilities $50 \mathrm{~m}$ before the tunnel entrances and $50 \mathrm{~m}$ after the tunnel exits.

\section{Conclusions}

Based on the analysis of the impact laws of freeway tunnel groups in mountainous areas on the pupil diameter variations of drivers, the following conclusions are drawn, and suggestions are proposed:

(1) The analysis results of the nontunnel segment, continuous tunnel segment, and single tunnel segment demonstrate that the continuous tunnel segment had the greatest impact on the PPDV of drivers. The mean and standard deviation of the
PPDV of the drivers in the continuous tunnel section were greater than those in the single tunnel section and nontunnel section. Additionally, the fluctuation of the corresponding indicators was the largest and remained at a high level in that section; this should be considered in the design, construction, and operation management of tunnel groups.

(2) Based on the impact of tunnel groups on the change in the pupil diameter of drivers, a tunnel group can be defined as a continuous tunnel section with three or more tunnels and tunnel spacing of no more than $700 \mathrm{~m}$. The impact range of tunnel groups on the pupil diameter of drivers was determined as $225 \mathrm{~m}$ before entering the tunnel group to $350 \mathrm{~m}$ after exiting the tunnel group. 
(3) Lighting transition zones should also be established at the exit zones of tunnels to improve the continuity of the driving environment.

(4) There was a negative correlation between the PPDV and the lengths of the connecting zones in the tunnel groups. Shorter connecting zones resulted in a higher PPDV and vice versa. The critical safety length of the connecting zone in the tunnel groups was determined as $L_{C}=100 \mathrm{~m}$. When $L_{C} \leq 100 \mathrm{~m}$, it is recommended that environmental improvement facilities be established for all the connecting zones. When $L_{C}>100 \mathrm{~m}$, it is recommended to establish environmental improvement facilities $50 \mathrm{~m}$ before the tunnel entrances and $50 \mathrm{~m}$ after the tunnel exits.

In this study, the Wen-Ma section of G4217 Rong-Chang Freeway was used as the experimental scene to obtain relevant data, and the impact of tunnel groups on the pupil changes of drivers was analyzed. However, there are some limitations to this study. Firstly, this experiment is performed during the daytime and the corresponding data are obtained. Different research results may be obtained if the same experiment is repeated at nighttime. Secondly, it must be noted that in an actual driving behavior adaptation process, the tunnel group environment may also affect other psychological and physiological indicators of drivers, such as the heart rate and fixation. Therefore, the nighttime experiment and the effects of tunnel groups on other factors of drivers should be further investigated.

\section{Data Availability}

The data used to support the findings of this study are available from the corresponding author upon request.

\section{Conflicts of Interest}

The authors declare that they have no conflicts of interest regarding the publication of this study.

\section{Acknowledgments}

This work was supported by The Science and Technology Plan Project of Department of Transportation of Sichuan Province/Research on Design and Control Index system of long and continuous Steep Slope of Freeway based on Operation Safety (grant number 2018-A-04).

\section{References}

[1] L. W. Gan, Z. Q. Chen, W. B. Yang, and G. Y. Ma, "Hazard identification and analysis of large-scale tunnels under complex geological conditions," Subgrade Engineering, vol. 201, no. 6, pp. 69-74, 2018.

[2] H. M. Wang, L. Lan, C. He, and Z. Zheng, "Study on truck driving risk and countermeasures of super-long downhill tunnel group of expressway," Highway, vol. 65, no. 04, pp. 237-242, 2020.

[3] W. H. Zhao, H. X. Liu, W. Liu, and T. Zhu, "Visual features of driver in exit and entrance of tunnel group," Journal of
Transport Science and Engineering, vol. 27, no. 3, pp. 75-81, 2011.

[4] T. Tan, Y. C. Wang, and C. H. Zong, "Research on the difference of drivers' visual characteristics in different zones of highway tunnel group," Logistics Science and Technology, vol. 10, pp. 68-72, 2020.

[5] L. Wu, H. X. Liu, and T. Zhu, "Study on the variation law of driver's visual characteristics and safety state discriminant model of highway long tunnel," Highway, vol. 061, no. 001, pp. 138-143, 2016.

[6] Y. F. Zhao, S. R. Zhang, and H. Wei, "Operating speed characteristics of tunnel group sectionHow can you not see my pain?," Journal of Chang'an Universityn, vol. 32, no. 6, pp. 67-72, 2012.

[7] S. Fang, H. Wu, J. Liao, and F. Wang, "Safety evaluation of freeway tunnel groups in mountainous areas," Journal of Tongji University, vol. 41, no. 5, pp. 693-699, 2013.

[8] J. Wang, A. Pervez, Z. Wang, C. Han, L. Hu, and H. Huang, "Crash analysis of Chinese freeway tunnel groups using a fivezone analytic approach," Tunnelling and Underground Space Technology, vol. 82, no. DEC, pp. 358-365, 2018.

[9] Y. Ma and R. Fu, "Research progress of drivers' visual characteristics and driving safety," China Journal of Highway and Transport, vol. 6, pp. 82-94, 2015.

[10] Y. Yan, F. Ye, X. F. Wang, and H. Hu, "Analysis of environmental illumination and driver pupil area in tunnel group zones," Journal of South China University of Technology, vol. 12, pp. 89-96, 2016.

[11] C. He, Y. Fang, Y. Zeng, and Y. Zhang, "Intelligent control of ventilation, lighting and disaster prevention for highway tunnel group or abut tunnels," in Proceedings of the Second International Conference on Transportation Engineering, Chengdu, China, July 2009.

[12] W. J. Zhu and X. D. Pan, "Critical driving speed research on tunnel group connection segments," Journal of Transportation Engineering \& Information, vol. 12, no. 1, pp. 74-78, 2014.

[13] K. Yang and C. Guo, "Traffic safety of highway adjoining tunnel and tunnel group in accidents," in Proceedings of the Second International Conference on Transportation Engineering, Chengdu, China, July 2009.

[14] J. L. Mei, Z. G. Du, H. R. Zheng, and L. B. Yang, "Research on visual load at entrance area of extra-long tunnel in different periods," China Safety Science Journal, vol. 31, no. 6, pp. 176-181, 2021.

[15] X. L. Wu, Q. Zhang, and Y. Pan, "Extra-long tunnel special light contrast experimental study on the environment," Technology of Highway and Transport, vol. 37, no. 3, pp. 125-131, 2021.

[16] S. Wang, Z. Du, G. Chen, H. Zheng, Z. Tang, and F. Jiao, "Drivers' visual characteristics in small-radius optically long tunnels on rural roads," Tunnelling and Underground Space Technology incorporating Trenchless Technology Research, vol. 113, pp. 1-11, 2021.

[17] H. Huang, Y. Peng, J. Wang, Q. Luo, and X. Li, "Interactive risk analysis on crash injury severity at a mountainous freeway with tunnel groups in China," Accident Anal Prevention, vol. 111, pp. 56-62, 2017.

[18] Y. Li, M. Wang, and H. Shi, "A study on section division of traffic accidents in expressway tunnel group," Chinese Journal of Underground Space and Engineering, vol. 8, no. s1, pp. 1591-1594, 2012.

[19] Y. C. Zhang, C. He, Y. Fang, and Y. H. Zeng, "Analysis on risk causation of traffic accidents in expressway tunnel group," China Safety Science Journal, vol. 9, no. 14, 2009. 
[20] A. Pervez, H. L. Huang, J. Y. Lee, C. Han, J. Wang, and $\mathrm{X}$. Zhang, "Crash analysis of expressway long tunnels using a seven-zone analytic approach," Journal of Transportation Safety \& Security, vol. 13, no. 1, pp. 1-15, 2019.

[21] A. Pervez, H. L. Huang, C. Y. Han, J. Wang, and Y. Li, "Revisiting freeway single tunnel crash characteristics analysis: a six-zone analytic approach," Accident Analysis \& Prevention, vol. 142, pp. 2-10, 2020.

[22] S. Y. Jung and X. Qin, "A data-driven approach to strengthening policies to prevent freeway tunnel strikes by motor vehicles," Accident Analysis \& Prevention, vol. 157, pp. 1-10, 2021.

[23] S. Hu, "Traffic accident characteristics and mechanism analysis of mountainous highway tunnel group," Highway, vol. 5, pp. 134-138, 2016.

[24] M. M. Duan, B. M. Tang, X. H. Hu, B. K. He, and T. Z. Liu, "Visual load of visual load of tunnel entry and exit of high tunnels," Transportation System Engineering and Information, vol. 18, no. 03, pp. 117-123, 2018.

[25] Z. G. Du and H. S. Strictly, "Highway tunnel share shard and sharp: dark adaptation time based on pupil area change," Highway Traffic Technology, vol. 30, no. 05, pp. 98-102, 2013.

[26] Ministry of Transport of the People's Republic of China, Specifications for Design of Highway Tunnels Section, Ministry of Transport of the People's Republic of China, Beijing, China, 2014.

[27] F. Ye and B. Gong, "Study on the visual light and shade adaptation characteristics of tunnel groups based on pupil changes," Highway and Automobile Transportation, vol. 000, no. 002, pp. 40-44, 2018.

[28] S. Z. Jiang, X. G. Zhang, K. Wang, and J. P. Gao, "Research on visual recognition characteristics of gate-type signs on mountainous expressway," Science Technology and Engineering, vol. 19, pp. 294-298, 2016.

[29] Z. F. Liu, R. Fu, and Y. Ma, "Driver's minimum visual attention demand under high-speed car-following condition," China Journal of Highway and Transport, vol. 031, no. 004, pp. 28-35, 2018.

[30] S. F. Wang, "Discussion on the classification of highway tunnel and the concept of highway tunnel group," Highway Tunnel, vol. 66, no. 2, pp. 10-14, 2009.

[31] C. Guo, M. Wang, and Y. Lu, "Intelligent traffic safety control technology of highway adjoining tunnel and tunnel group," in Proceedings of the Fourth International Conference on Transportation Engineering, pp. 1957-1962, Chengdu, China, October 2013.

[32] Z. W. Zhou, J. X. Ma, and T. Tan, "Evaluation of drivers' visual stability in highway adjacent tunnel group sections," Forest Engineering, vol. 36, no. 4, pp. 116-122.

[33] M. K. Chen, J. X. Ma, and T. Lu, "Visual characteristics of drivers for driving through freeway tunnel," Traffic Information and Safety, vol. 37, no. 3, pp. 86-92, 2019.

[34] S. H. Ather, "Catastrophe theory in work from heartbeats to eye movements," Biological Cybernetics, vol. 115, no. 1, pp. 39-41, 2021. 